\title{
Time-Resolved X-ray Studies Using Third Generation Synchrotron Radiation Sources. *
}

\author{
$\mathrm{ANL} / \mathrm{CP}--72328$ \\ Dennis M. Mills \\ DE $92 \quad 003516$ \\ Advanced Photon Source \\ Argonne National Laboratory \\ 9'700 South Cass Avenue \\ Argonne, IL 60439
}

October, 1991

$\mathrm{cz}$

The submitted manuscript has been authored
by a contractor of the U.S. Government
under contract No. W.31-109ENG.38.
Accordingly. the U. S. Government retains a
nonexclusive, royalty.free license to publish
or reproduce the published form of this
contribution, or allow others to do so, for
$U$.S. Government purposes.

*This work supported by the U.S. Department of Energy, BES-Materials Sciences, under contract no. W-31-109-ENG-38

\section{DISCLAIMER}

This report was prepared as an account of work sponsored by an agency of the United States Government. Ne،ther the United States Government nor any agency thereof, nor any of their employees, makes any warranty, express or implied, or assumes any legal liability or responsibility for the accuracy, completeness, or usefulness of any information, apparatus, product, or process disclosed, or represents that its use would not infringe privately owned rights. Reference herein to any specific commercial product, process, or service by trade name, trademark, manufacturer, or otherwise does not necessarily constitute or imply its endorsement, recommendation, or favoring by the United States Government or any agency thereof. The views and opinions of authors expressed herein do not necessarily state or reflect those of the United States Government or any agency thereof. 


\title{
Time-Resolved X-ray Studies Using Third Generation Synchrotron Radiation Sources
}

\author{
Derinis M. Mills \\ Advanced Photon Source \\ Argonne National Laboratory \\ 9700 S. Cass Avenue \\ Argonne, IL USA 60439
}

\begin{abstract}
The third generation, iligh-brilliance, hard $x$-ray, synchrotron radiation (SR) sources currently under construction (ESRF at Grenoble, France; APS at Argonne, Illinois; and SPring-8 at Harima, Japan) will usher in a new' era of $x$-ray experimentation for both physical and biological sciences. One of the most exciting areas of experimentation will be the extension of $x$-ray scattering and diffraction techniques to the study of transient or time-evolving systems. The high repetition rate, short-pulse duration, high brilliance, and variable spectral bandwidth of these sources make them ideal for $x$-ray time-resolved studies.

The temporal properties (bunch length, interpulse period, etc.) of these new sources will be summarized. Finally, the scientific potential and the technological challenges of time-resolved $x$-ray scattering from these new sources will be described.
\end{abstract}

\section{Introduction}

The unique properties of synchrotron radiation, particularly, the high flux and temporal modulation, naturally lend it for use in time-resolved $x$-ray studies. Over the last several years, the kinetics of a variety of interesting transient physical systems with time scales spanning from hours to nanoseconds have been explored using radiation emitted from storage ring sources. Both the scientific and technical aspects of many of these time-resolved experiments have been chronicled in several recent review articles and/or workshop proceedings [1-4], and the reader is referred to these existing papers for a more detailed account of research in this expanding field. Hence, the content of this presentation will focus on the advances in the field of time-resolved studies that might arise with the advent of the next (third) generation of storage ring sources currently being constructed in Europe, Japan, and the United States. The discussion here will be limited to sources designed to optimize radiation output in the hard $x$-ray region (i.e., $E \geq 10 \mathrm{keV}$ ).

\section{Third Generation Sources}

Third generation synchrotron radiation sources can be characterized as dedicated, low-emittance storage rings optimized for high brilliance $x$-ray beams (see Fig. 1). Put another way, these storage rings have been carefully designed to provide numerous, long, straight sectioris for insertion devices (wigglers and undulators) and a particle biam with a small source size and divergence. Three such storage ring sources are currently in various stages of completion: the European 
Synchrotron Radiation Facility (ESRF) in Grenoble, France: the Super Photon Ring (SPring-8) in Harima, Japan; and the Advanced Photon Source (APS) in Argonne, Illinois, USA. Although the details of each of these storage rings are different, all have similar design goals and specifications. I will use the specifications for the APS as archetypical of a third generation synchrotron source because I am most familiar with them: I will also attempt to point out major differences between the sources if relevant to time-resolved studies. (Detailed specifications of the radiation properties of these new sources can be found in references [5], [6] and [7].) The storage ring is specifically designed to accommodate a large number $(>30)$ of long (up to 5 meter) insertion devices. Particle beam emittances are approximately 1 nanometer-radian in the vertical and 10 nanometer-radian in the horizontal; the vertical emittance being determined by the need to be approximately equal to the natural vertical opening angle of the first harmonic of the undulator, typically 10 15 microradians. The third generation sources have particle beam energies in the range from 6 to $8 \mathrm{GeV}$. This beam energy is required to produce $x$-rays in the 4 to $40 \mathrm{keV}$ range from the first and third harmonics of planned undulators. A byproduct of this high particle energy is a high flux of very hard $x$-rays (E>50 KeV) produced from high field wigglers installed in straight sections. Because of the beam energy required, the circumference of these rings is large, 1104 meters in the case of the APS, corresponding to relatively long orbital periods $(3.68$ microseconds at the APS). However, that will not be the repetition rate of the radiation under normal operating conditions. In order to achieve the design current of 100 milliamperes while maintaining the low emittance, multi-bunch operation will be required. At this time, the APS plans to achieve this current level with 20 equally spaced bunches (184 nanosecond spacing), while the ESRF will attempt to accomplish this design goal with 992 bunches (2.8 nanosecond spacing). The typical bunch length for these sources will be 50-100 picoseconds (the APS calculated bunch length is 72 picoseconds FWHM).

\section{Time-Resolved Experiments on Third Generation Sources}

Flux increases of at least one order of magnitude over that at existing sources are expected from insertion devices on third generation sources through the $10 \mathrm{keV}$ to $100 \mathrm{keV}$ energy range (see Fig. 2). Clearly, an increase of this magnitude will make an impact on flux hungry experiments such as time-resolved $x$-ray diffraction or dispersive EXAFS and XANES. Increased ilux will allow smaller apertures to be used with those experiments where small beam sizes are required, for example, to examine traveling interfaces due to physical processes such as pressure waves, combustion fronts, thermal waves, etc.

The large number of hard $x$-rays generated from these sources may facilitate time-resolved experiments under extreme conditioris such as high pressure and high/low temperatures. High energy photons are particularly useful for these types of experiments in which the $x$-rays must pass through the walls of environmental cells (diamond anvil cells, furnaces, cryostats, elc.) to get to and from the sample. In addition, highly collimated beams may be required in order to illuminate the desired portion of the sample so that the parameters of interest (pressure, temperature, etc.) are relatively uniform throughout the volume of sample being probed. 
It is clear, however, that these new sources will have the greatest impact on those experiments that depend on beam brilliance. Increases in beam briliance four orders of magnitude greater than that available at existing synchrotron facilities are expected at the APS and equivalent sources. This improvement in beam brilliance should allow one to consider brilliance-limited experiments, which are currently difficult to perform in a static mode (such as high-resolution scattering experiments, surface scattering, ultra-small angle scattering, and microprobe experiments), in a time-resolved mode. In addition, high brilliance may also play an important role in flux-limited experiments since the well-collimated beams should reduce off-axis aberrations from optical components thereby facilitating bettei focusing performance for obtaining small beam sizes.

Although one generally associates high brilliance with the concept of more highly collimated photons on a sample, third generation sources will provide other interesting and useful radiation properties such as coherence. The applications of coherent $x$-ray beams to time-resolved studies may be prove to be very useful indeed. One such application is an extension of what is called in the visible region of the spectrum dynamic light scattering or intensity fluctuation spectroscopy [8]. In the visible portion of the electromagnetic spectrum, one can observe this phenomena via the so-called speckle patterns that result when coherent light is scattered. Using this effect, time-resolved studies of critical fluctuations near phase transitions, for instance, are investigated with visible light. The length scales that can be studied is limited by the wavelength of the probing light, which is several thousand Angstroms for visible light. Using $x$-rays, one could probe the dynamics of processes at atomic-length scales, opening up a host of new possibilities for time-resolved, equilibrium and non-equilibrium thermodynamic studies. The key to the success of this type of experime.nt is the production of an x-ray beam that is both transversely and longitudially coherent over the scattering volume of the sample. The third generation of high-brilliance $x$-ray solirces will have a considerable amount of coherent $x$-ray flux (see Fig. 3). Although only a fraction of the total number of photons emitted at that wavelength are coherent, there is ample flux for many types of time-resolved diffraction experiments. For instance, on an APS undulator nearly $10^{10}$ photons/sec-0.1\%bw of transversely coherent photons will be emitted near $8 \mathrm{keV}$. [The longitudinal coherence length, $L_{c}$, of the radiation is determined by the monochromaticity of the beam: $L_{C}=(\lambda / 2)(\lambda / \Delta \lambda)$.]

None of the experiments mentioned above make direct use of the modulated time structure of the radiation. One of the more interesting proposed applications of the pulsed nature of the radiation is its use to capture transient phenomena in large macromolecular crystals. In particular, if it were possible to obtain a usable diffraction pattern from a crystal with the x-rays from one bunch of particles, then the temporal resolution of such a technique could be less than 100 picoseconds. Since it is clear that a crystal could not be rotated over reasonable angles on this timescale, monochromatic techniques that use angle integrating methods are not appropriate. An alternative to the monochromatic technique is a polychromatic Laue method in which a wavelength integration is performed by the diffracting planes. Such an approach is ideal for time-resolved experiments. It has been calculated that between $10^{10}$ and $10^{13}$ photons are required to produce a interpretable Lave photograph [9]. If a broad-energy bandwidth input beam is used, say 5-15 keV, 
then, in this bandwidth from one burst of $x$-rays from a wiggler on the APS running with 5 ma per bunch, approximately $4 \times 10^{10}$ photons/horizontal milliradian will be emitted. By collecting over the full horizontal extent of the wiggler beam and slightly increasing the bandwidth, this number could be increased to over $10^{11}$ photons per burst. Several years ago, the feasibility of such experiments were explored using an APS-prototype undulator installed in the Cornell Electron/Position Storage Ring (CESR) [10]. Single-pulse diffraction patterns were collected, although not in a time-resolved mode nor with the necessary quality to permit new structural information to be extracted.

\section{Summary}

Although the third generation of synchrotron radiation sources will provide unparalleled opportunities for researchers interested in time-resolves studies, there are still many technological and experimental challenges that need attention. At the risk of being too parochial, I will mention the work the APS staff has been doing to address some of the important instrumentation issues. Obviously, one critical area is detector development. There are classes of time-resolved experiments that are already constrained by detec or limitations with currently operating sources. New sources will only exacerbate the situation. Development of high-count-rate and fast-framing one-and two-dimensional detectors will be crucial to the success of many proposed time-resolved experiments. A programmable Charge Coupled Device (CCD) detector has been developed by the staff of the APS that allows operation in a "streak camera" mode (Fig. 4) permitting 1-D frame transfers to be completed in approximately 2 microseconds [11]. This is an important milestone since it means that, when the storage ring is run in singlebunch mode (as may be required for some timing experiments), a frame transfer can be completed before the next burst of $x$-rays arrives. Thus, the temporal resolution jumps from 2 microseconds to the duration of the burst itself, approximately 100 picoseconds. We have just begun to investigate two-dimenzional arrays that have similar framing rates.

As mentioned above, there may be the need to run in a special timing mode (i.e., one-bunch mode) for certain types of experiments. Because it is expected that only 5 milliamperes of current will be in a single bunch, timing modes are usually not appreciated by the other users because the current in the storage ring and, hence, the fiux and brilliance are reduced to a small fraction of the usual value: $5 \%$ in the example cited here. To lessen the effect of running in a timing mode on other users not interested in timing experiments, we are looking into possible techniques of altering the natural time structure of the ring in a local way (i.e., on selected beamlines) by using high speed mechanical choppers and modulating mirrors and monochromators [12]. Single pulses have been successfully isolated with mechanical choppers on large storage rings such as CESR [13] where the interpulse period is long (2.56 microseconds). We hope to pursue this approach further to see if it is applicable to the APS when more than one bunch of stored particles are in the ring.

Perhaps the most daunting hurdle, however, is uniform (spatial and temporal) excitation of the sample. This problem, however, is not unique to time- 
resolved studies involving synchrotron radiation, and, fortunately, one can borrow techniques from other disciplines (such as time-resolved laser techniques) to overcome this challenge. Certainly the next decade, as the ESRF, SPring-8, and APS all become fully operational, will be an exciting and stimulating time for those interested in the study of transient phenomena using $x$-rays.

\section{Acknowledgements}

The author would like to thank Prof. Mark Sutton, McGill University, and Dr. Brian Stephenson, IBM, for helpful discussions about their work on $x$-ray intensity fluctuation spectroscopy and Drs. Brian Rodricks, Dean Haeffner, and Wen Bing Yun, APS, who supplied the figures in this text. This work is supported by the U.S. Department of Energy, BES-Materials Science, under Contract No. W-31-109ENG-38.

\section{References}

[1] S. Gruner, Science 23 8, p 305, 1987.

[2] "Time-Resolved Studies and Ultra-FastDetectors: Workshop Report," R. Clarke, P. Sigler, and D. M. Mills, ANLAPS-TM-2, Argonne National Laboratory, Argonne, Illinois, USA.

[3] B. C. Larson and J. Z. Tischler, Advanced X-ray/EUV Radiation Sources and Applications, San Diego, CA, SPIE Proceedings 134 5, p 90,1990.

[4] "Time-Resolved Studies," D. M. Mills, Handbook on Synchrotron Radiation, Vol. 3, G. Brown and D. E. Moncton, eds., Elsevier Science Publishers, B. V., p 291, 1991.

[5] "The Red Book," European Synchrotron Radiation Facility, B.P. 20038043 Grenoble Cedex, France.

[6] "Characteristics of the 7-GeV Advanced Photon Source: A Guide for Users," G. K. Shenoy, P.J. Viccaro, and D. M. Mills, ANL-88-9, Argonne National Laboratory, Argonne, Illinois, USA.

[7] "SPring-8 Project," The JAERI-RIKEN SPRing-8 Project Team, 2-28-8 Honkomagome, Bunkyo-ku. Tokyo, Japan.

[8] M. Sutton, S. G. J. Mochrie, T. Greytak, S. E. Nagler, L. E. Berman. G. A. Held, and G. B. Stephenson, Nature 35 2, p 608, 1991.

[9] I. J. Clifton, V. Fulop, A. Hadfield, P. Norlund, I. Andersson, and J. Hajdu, Nucl. instrum. and Meth. A 303, p 476, 1991.

[10] D. M. E. Szebenyi, D. Bilderback, A. LeGrand, K. Moffat, W. Schildkamp, and T.-Y. Teng, Trans. Am. Cryst. Soc. 2 4, p 167, 1988. 
[11] B. Rodricks and C. Brizard, Nucl. Instrums. and Meth., in press.

[12] D. M. Mills, Rev. Sci. Instrum. 6 0, p 2338, 1989.

[13] A. LeGrand, W. Schildkamp, and B. Blank, Nucl. Instrum. and Meth. A 275, p $442,1989$.

\section{Figure Captions}

Fig.1 Plot of $x$-ray brilliance as a function of $x$-ray energy for the Advanced Photon Source and several existing sources currently operating in the United States.

Fig.2 A plot of the $x$-ray flux as a function of $x$-ray energy for one of the wigglers planned for the Advanced Photon Source and several existing sources currently operating in the United States.

Fig.3 A plot of the number of transversely (spatially) coherent photons as a function of energy for one of the proposed undulators to be placed on the Advanced Photon Source.

Fig.4 Schematic of the experimental arrangement for operating a CCD detector in a one dimensional fast timing mode. An x-ray opaque mask covering all pixels except the top row is placed between the detector and $x$-rays. The top, exposed, row of pixels is illuminated by the $x$-rays for a prescribed amount of time and then parallel transferred to the adjacent row behind the mask. This transfer time can occur in several microseconds. The pixels rows behind the mask are used as storage until the entire CCD array is filled, at which time it is read out serially. 


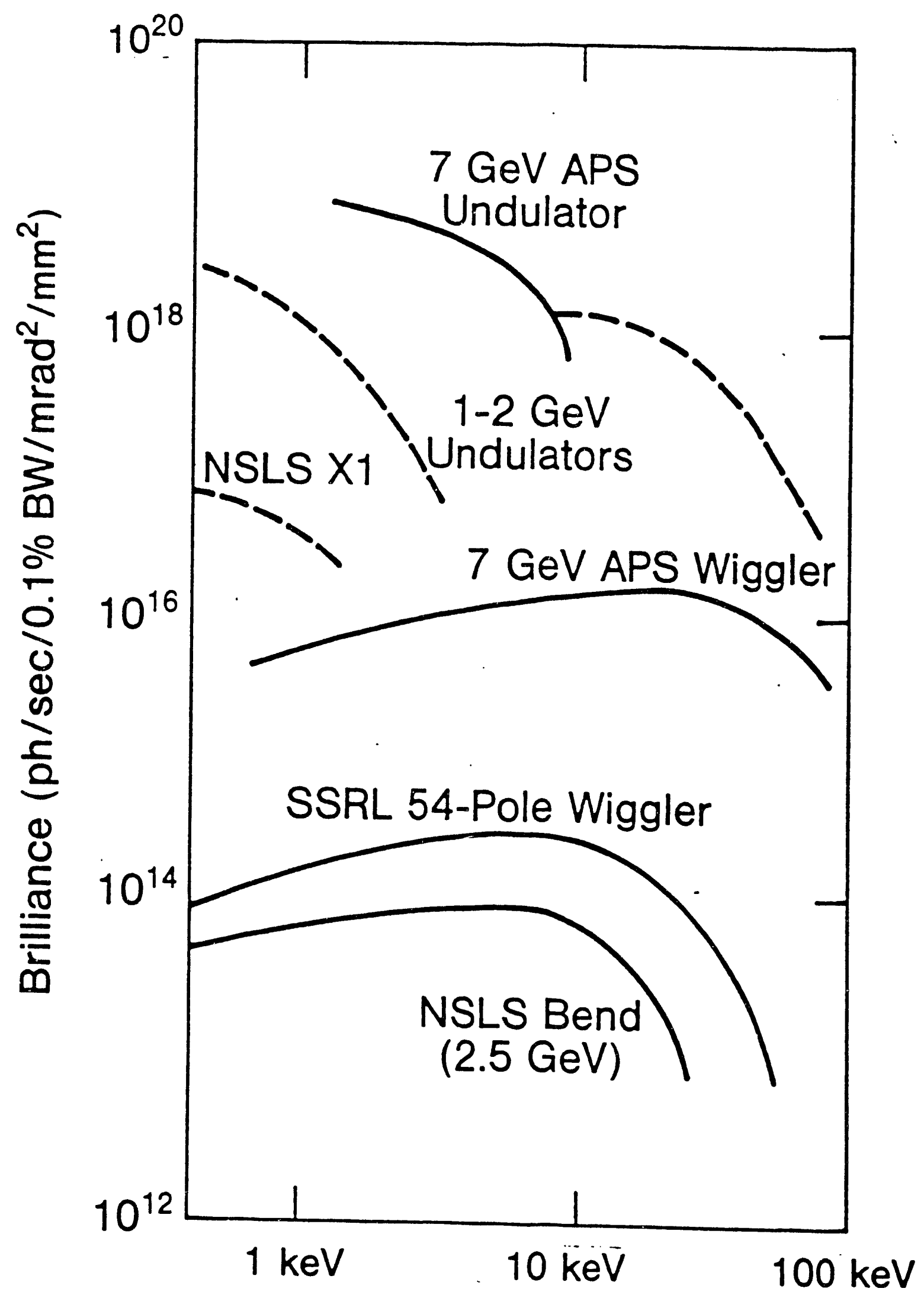

Figure 1 


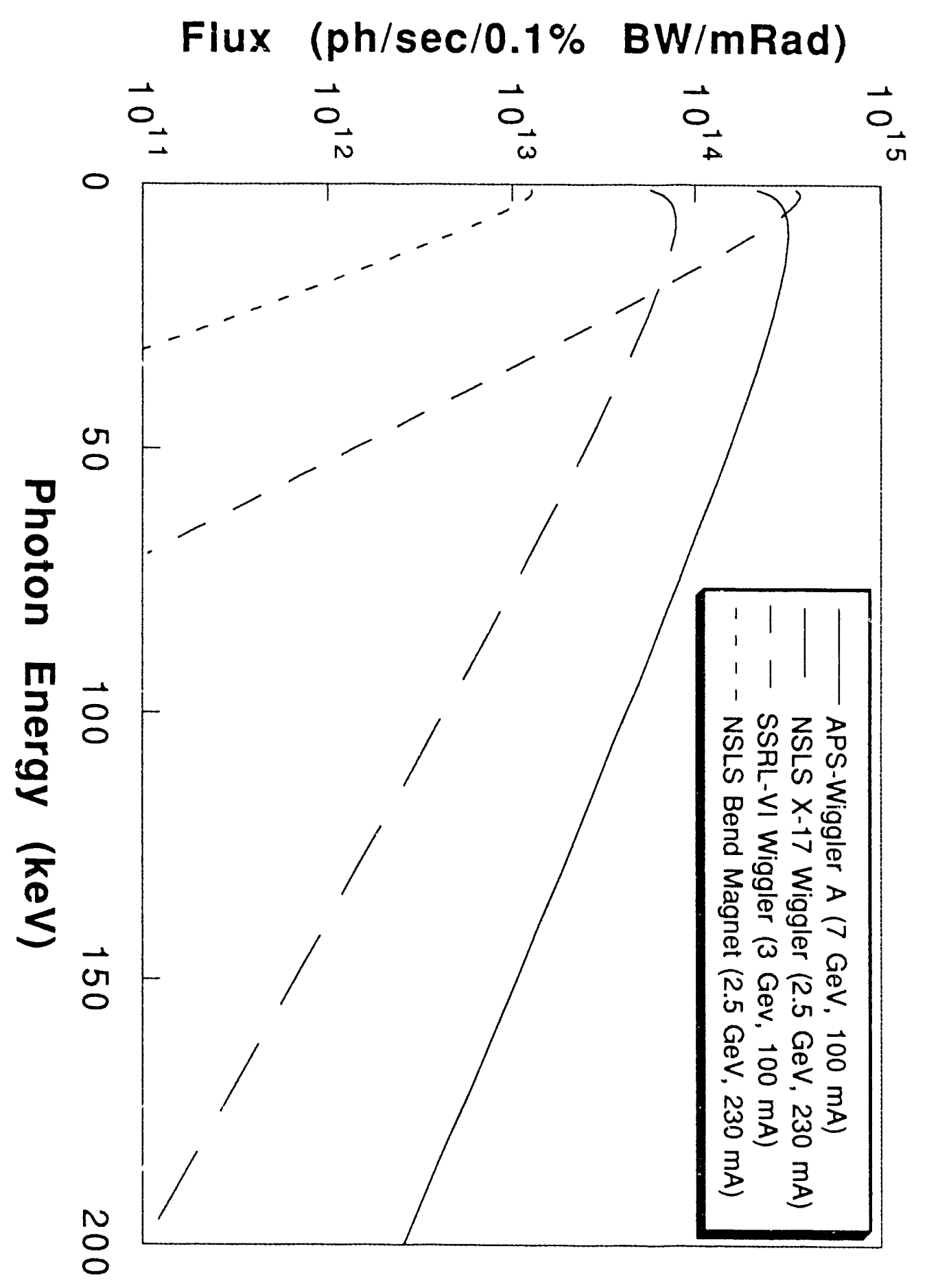

Figure 2 


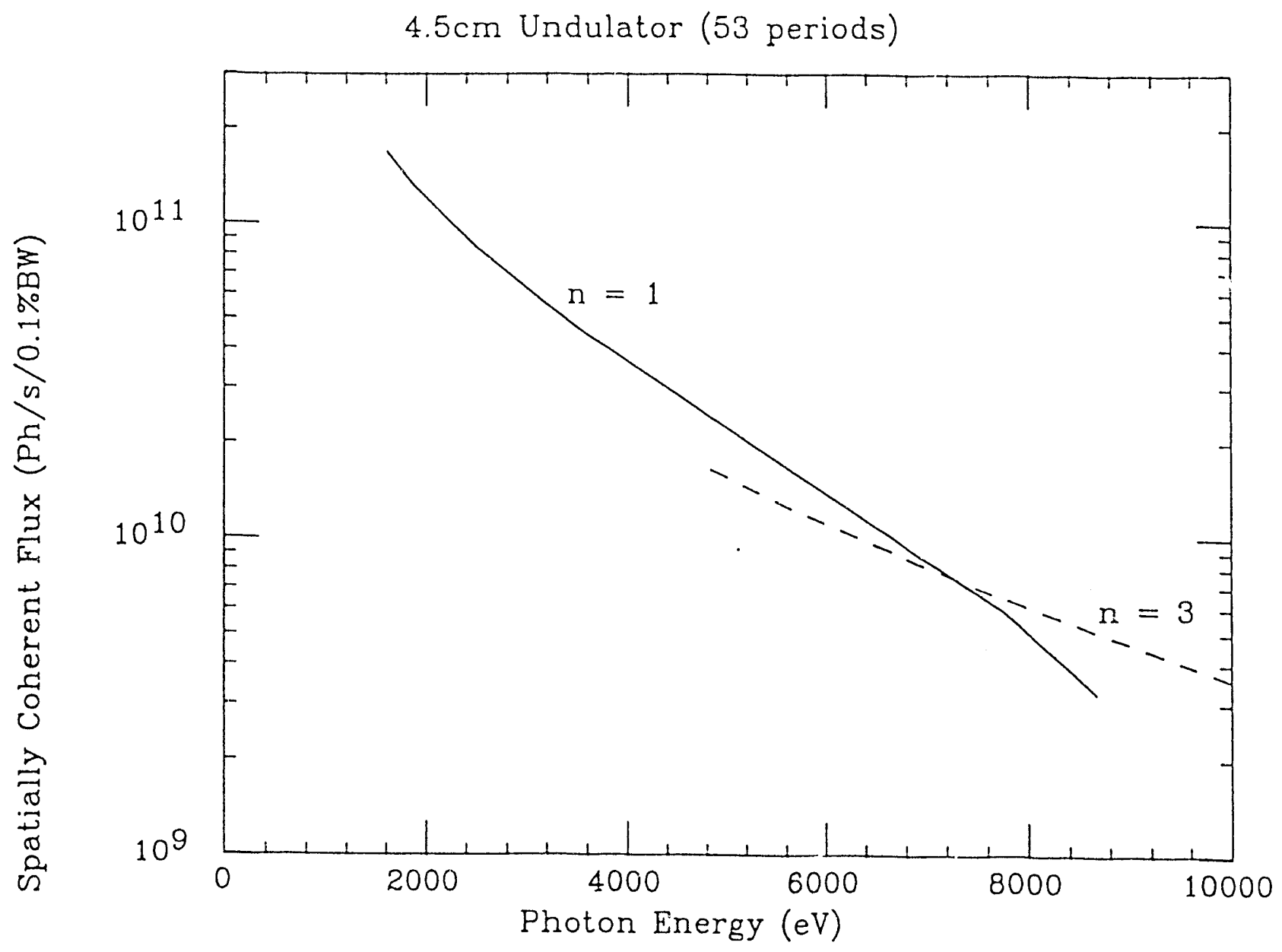

Figure 3 


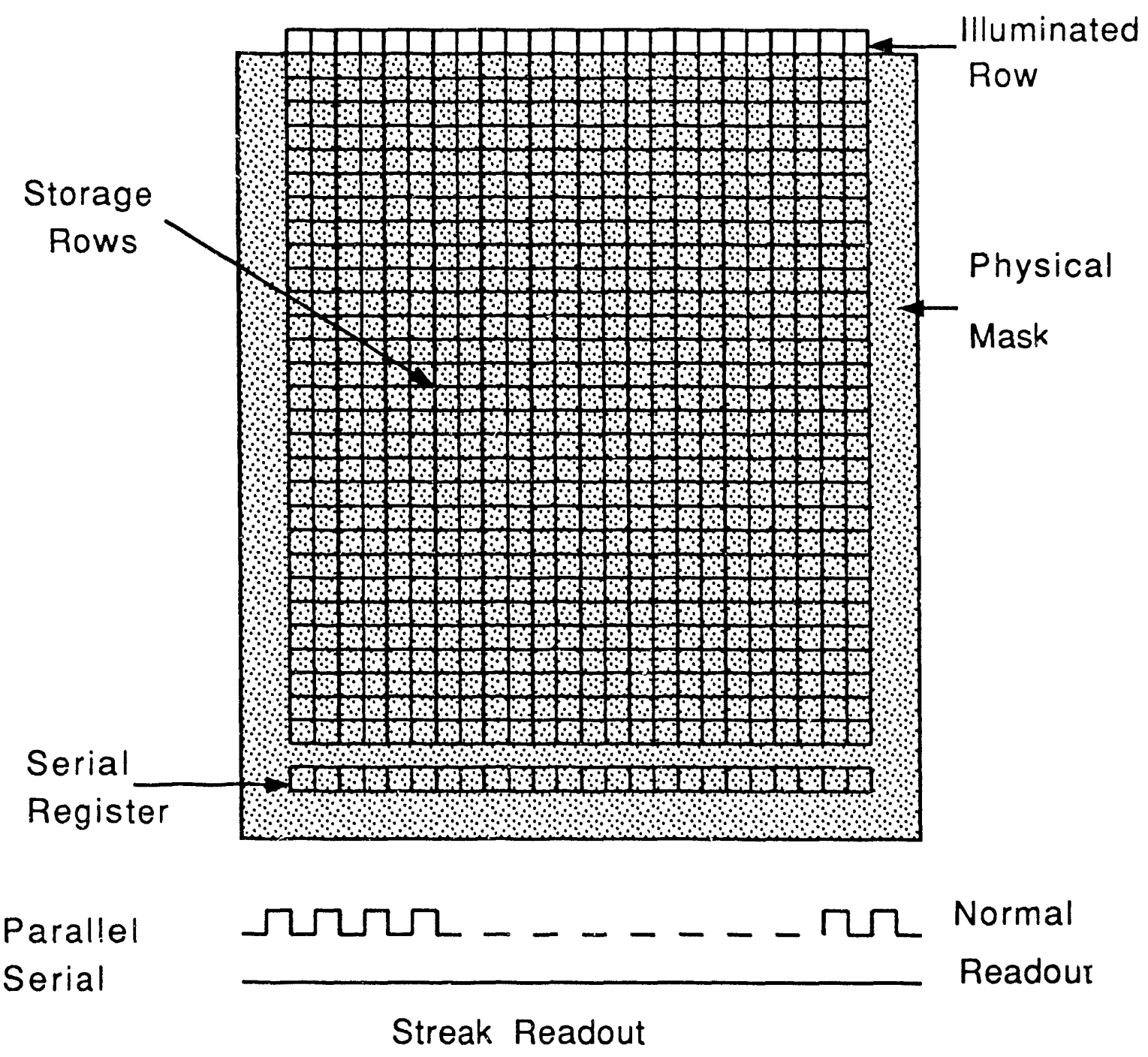

Figure 4 

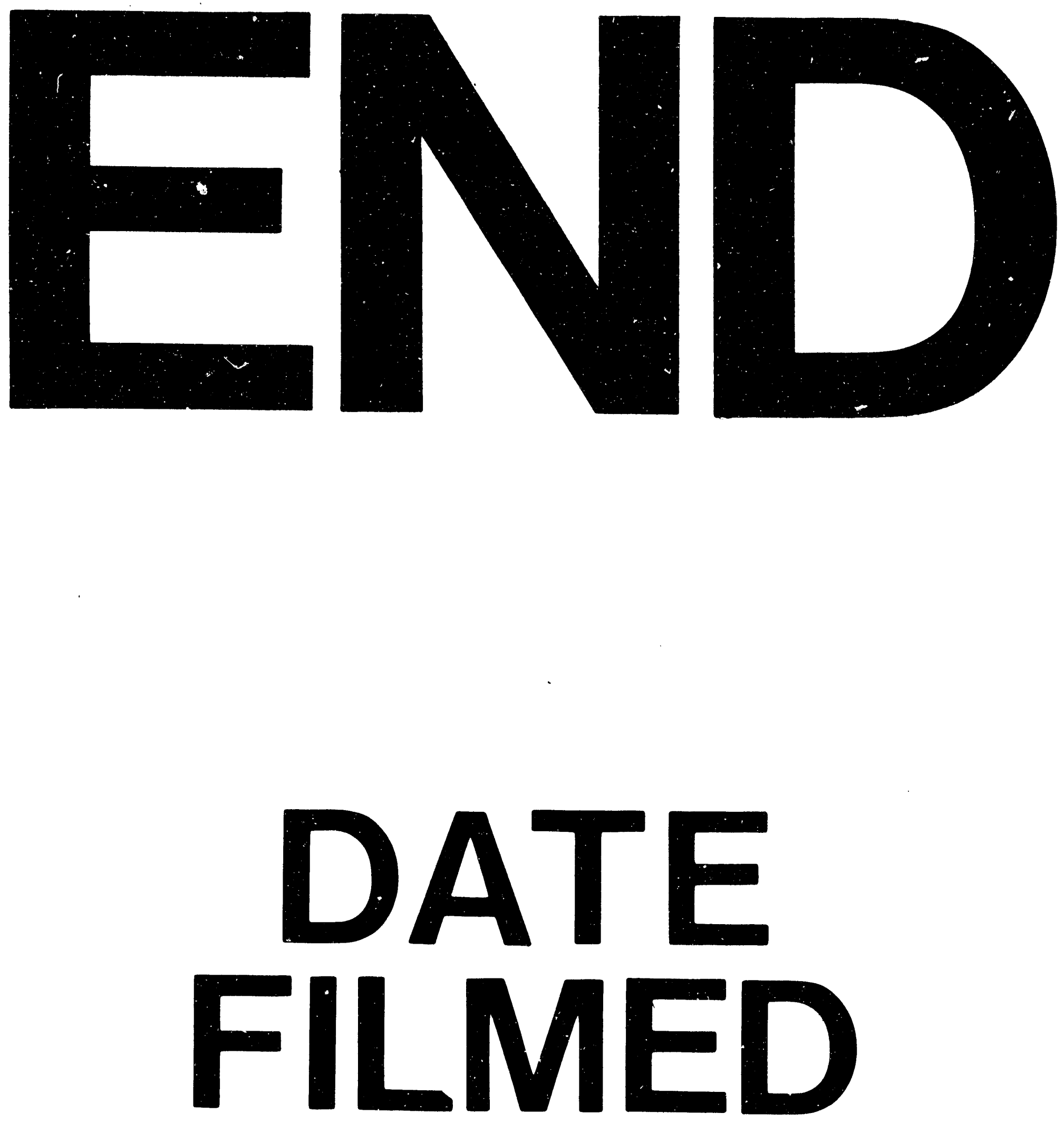

1

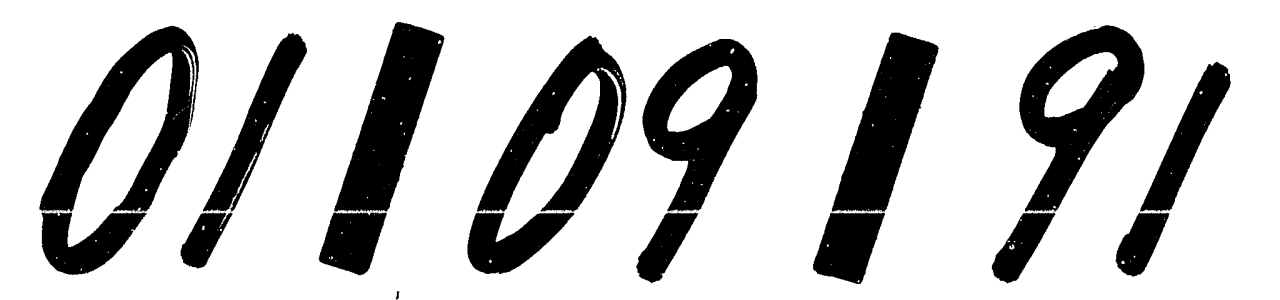

